\title{
Molecular cloning of metal-responsive transcription factor-1 (MTF-1) and transcriptional responses to metal and heat stresses in Pacific abalone, Haliotis discus hannai
}

\author{
Sang Yoon Lee and Yoon Kwon Nam*
}

\begin{abstract}
Background: Metal-responsive transcription factor-1 (MTF-1) is a key transcriptional regulator playing crucial roles in metal homeostasis and cellular adaptation to diverse oxidative stresses. In order to understand cellular pathways associated with metal regulation and stress responses in Pacific abalone (Haliotis discus hannai), this study was aimed to isolate the genetic determinant of abalone MTF-1 and to examine its expression characteristics under basal and experimentally stimulated conditions.

Results: The abalone MTF-1 shared conserved features in zinc-finger DNA binding domain with its orthologs; however, it represented a non-conservative shape in presumed transactivation domain region with the lack of typical motifs for nuclear export signal (NES) and Cys-cluster. Abalone MTF-1 promoter exhibited various transcription factor binding motifs that would be potentially related with metal regulation, stress responses, and development. The highest messenger RNA (mRNA) expression level of MTF-1 was observed in the testes, and MTF-1 transcripts were detected during the entire period of embryonic and early ontogenic developments. Abalone MTF-1 was found to be Cd inducible and highly modulated by heat shock treatment.

Conclusion: Abalone MTF-1 possesses a non-consensus structure of activation domains and represents distinct features for its activation mechanism in response to metal overload and heat stress. The activation mechanism of abalone MTF-1 might include both indirect zinc sensing and direct de novo synthesis of transcripts. Taken together, results from this study could be a useful basis for future researches on stress physiology of this abalone species, particularly with regard to heavy metal detoxification and thermal adaptation.
\end{abstract}

Keywords: Abalone, Haliotis discus hannai, MTF-1, Heavy metal, Heat shock

\section{Background}

Metal-responsive transcription factor-1 (MTF-1; also termed metal-regulatory transcription factor-1 or metalresponsive-element-binding transcription factor-1) is a key transcriptional regulator playing pivotal roles in metal homeostasis and detoxification (Laity and Andrews 2007; Günther et al. 2012a). In addition to its fundamental role for homeostatic metal regulation, this multitasking

\footnotetext{
* Correspondence: yoonknam@pknu.ac.kr

Department of Marine Bio-Materials \& Aquaculture, Pukyong National University, Busan 48513, South Korea
}

transcription factor has also been known to be closely involved in cellular adaptation and protection against oxidative stresses through regulating the transcription of diverse genes related with host defense-related pathways (Günther et al. 2012a; Lichtlen and Schaffner 2001). They include metal reservation/detoxification (e.g., metallothionein, MT; the main target of MTF-1), metal ion transport (e.g., $\mathrm{Zn}$ or $\mathrm{Cu}$ transporters), iron homeostasis/anti-microbial responses (e.g., hepcidin), cellular redox homeostasis (e.g., selenoproteins and thioredoxin reductase), and glutathione biosynthesis 
(e.g., glutamate cysteine ligase) (Günther et al. 2012a; Lichtlen et al. 2001; Stoytcheva et al. 2010).

In a structural viewpoint, MTF-1 has been considered as a conserved transcription factor to possess six $\mathrm{C}_{2} \mathrm{H}_{2}$ type zinc fingers as the DNA-binding domain to recognize metal responsive elements (MREs) (Giedroc et al. 2001). As a cellular metal and stress sensor, the activity regulation of MTF-1 is generally characterized by the three successive steps, i.e., nuclear-cytoplasmic shuttling upon stress exposure, DNA-binding, and the interaction(s) with other coactivators to modulate the target gene transcription ( $\mathrm{Li}$ et al. 2008). To execute the transcriptional regulation, MTF-1 binds to the specific site, called MRE (core sequence $=$ TGCRCNC), in the promoter region of target gene (Günther et al. 2012a). Accordingly, the transcriptional expression of MTF-1 gene itself has been reported to be constitutive and not to be affected by heavy metal and other stressor treatments because its regulatory functions should be controlled mainly at post-translational levels (Auf der Maur et al. 2000; Bi et al. 2006). Structural scheme and functional context of MTF-1 above-described are believed to be widespread in the vertebrate lineage, although the majority of empirical information has come from mammalian MTF-1s.

However, in contrast to richness of knowledge on mammalian orthologs, molluscan MTFs have been narrowly explored barring only couples of previous reports (Qiu et al. 2013; Meng et al. 2015). Nevertheless, it is noteworthy that currently available molluscan MTF-1 sequences from public databases have suggested that mollusc species may show non-canonical features in their MTF-1 structures, which may differ from vertebrate orthologs. For instances, unlike vertebrate orthologs, molluscan MTF-1s often lack several typical motifs in presumed transactivation domains. Furthermore, a recent study has claimed that the nuclear-cytoplasmic shuttling, a key prerequisite step for vertebrate MTF-1s, might not always be an absolute precondition in certain mollusc species (Meng et al. 2015).

Pacific abalone, Haliotis discus hannai, is a highly valued seafood mollusc not only in Korea but also in other East-Asian countries. Intensive aquacultural operation for abalone farming using the marine net-cage system has been established in Korean aquaculture domain. During the last decade, the remarkable growth of abalone production in a quantitative term has been achieved (Park and Kim 2013). However, more recently, the sustainable progress of abalone culture has been considerably hurdled by the depressed productivity mainly in relation to frequent outbreaks of high mortality and physiological deformity in many abalone farms (Park and Kim 2013; Kang et al. 2015). Considering that abalone farming in Korea mainly relies on the net-cage facility installed in coastal areas, the heavy metals or other related pollutants contaminated in both water and sediments could be significant factors to provoke cellular toxicity and oxidative stress in farmed abalones (Kim et al. 2007).

However, despite its importance, adaptive or defensive functions to such environmental perturbations have been limitedly investigated in this abalone species, and almost no information has been available with respect to the coordinated regulations of genes involved in cellular pathways associated with metal regulation and oxidative stress responses (Kim et al. 2007; Lee and Nam 2016a). For this reason, understanding of MTF-1 from abalone species would be much useful to better comprehend orchestrated and coordinated regulations of host defense genes in this abalone species. Based on this need, this study, as a startpoint research, was aimed to characterize the genetic determinant of MTF-1, the superordinate regulator for diverse host defense genes, from the Pacific abalone $(H$. discus hannai). For this, we isolated and characterized the full-length complementary DNA (cDNA) encoding the abalone MTF-1, bioinformatically dissected its $5^{\prime}$-upstream regulatory region, and also scrutinized expression patterns of MTF-1 under both non-stimulated and stress-challenged (i.e., heavy metal exposure and heat shock) conditions.

\section{Methods}

Abalone samples and molecular cloning of MTF-1

Abalones ( $H$. discus hannai) used in this study were experimental stocks maintained at Experimental Fish Culture Station, Pukyong National University (PKNU), Busan, South Korea. Abalones were maintained with semi-water recirculation system equipped with 3-ton capacity of rectangular culture tanks, in which the tanks were connected with protein skimmers, custom-designed mechanical filters, and 1- $\mu \mathrm{m}$-mesh filter. Throughout the experiment, water temperature and dissolved oxygen were kept to be ranged within $20 \pm 1{ }^{\circ} \mathrm{C}$ and $8 \pm 1 \mathrm{ppm}$, respectively. Abalones were fed with frozen or dried seaweeds until 2 days before stress exposure treatments. Daily water exchange rate was about $20 \%$, and in-tank wastes including feces and debris on the bottom were removed twice every day.

Based on the NGS-transcriptome analysis of the juvenile abalone tissues (unpublished data), partial NGS clones representing the significant homology to known animal MTF-1s were selected and assembled into a contig. In order to get full-length cDNA version, rapid amplifications of cDNA ends (RACE) at both $5^{\prime}$ - and 3 '-directions were carried out using total RNA isolated from a whole body sample and SMARTer ${ }^{\oplus}$ RACE 5'/3' Kit (Clontech Laboratories Inc., Mountain View, CA, USA) according to the manufacturer's instructions. Oligonucleotide primers used in this study are listed in Additional file 1: Table S1. The amplified fragments were 
sequenced and again subjected to contig assembly. Based on the assembled sequence in a contig, full-length abalone MTF-1 cDNA was re-isolated by RT-PCR amplification using the same total RNA aforementioned. Amplified RTPCR products were directly sequenced at both forward and reverse directions to obtain a representative cDNA sequence for abalone MTF-1.

From the cDNA sequence, the $5^{\prime}$-upstream region of abalone MTF-1 gene was cloned by genome walking method. Using the genomic DNA prepared from an individual muscle, genome walking to $5^{\prime}$-upstream region was conducted with designated pairs of gene-specific primers and Universal Genome Walker Kit (Clontech Laboratories Inc., USA) according to the manufacturer's instructions. Amplified fragments were TA cloned into $\mathrm{pGEM}^{\circ} \mathrm{T}^{\circ}$ easy vector (Promega, Madison, WI, USA), sequenced and assembled into a single contig. Afterward, the continuous, genomic fragment containing the $5^{\prime}$-flanking region was again PCR isolated from the genomic DNA template abovementioned and directly subjected to the sequencing to confirm the representative sequence of the abalone MTF-1 proximal promoter region.

\section{Bioinformatic sequence characterization}

With the ORF Finder program (https://www.ncbi.nlm.nih. gov/orffinder/), the open reading frame (ORF) of abalone MTF-1 was predicted and deduced amino acid sequence was obtained. Based on the homology search using NCBI BLASTx (http://blast.ncbi.nlm.nih.gov/Blast.cgi), sequence homology of abalone MTF-1 with its orthologs was examined. Parameter scores for the primary structure of MTF-1 were estimated using ExPASy ProtParam tool (http://web.expasy.org/protparam/). Multiple sequence alignment was done using CLUSTALW program (http:// www.genome.jp/tools-bin/clustalw). Identification of putative zinc finger domains was carried out with Simple Modular Architecture Research Tool (SMART; http://smart.embl.de/). Predictions of potential nuclear localization signal (NLS) and nuclear export signal (NES) were conducted with cNLS Mapper (http://nls-mapper.iab.keio.ac.jp/cgibin/NLS_Mapper_form.cgi) and NetNES 1.1 Server (http:// www.cbs.dtu.dk/services/NetNES/), respectively. The putative zinc finger-DNA binding regions from selected MTF-1 orthologs were subjected to molecular phylogenetic analysis using Molecular Evolutionary Genetics Analysis tool (ver. 7.0.21; http://www.megasoftware.net/). Putative transcription factor binding motifs in the abalone MTF-1 promoter were predicted with TRANSFAC ${ }^{\circ}$ software (http://genexplain.com/transfac; GeneXplain $\mathrm{GmbH}$, Wolfenbüttel, Germany).

\section{Tissue collection from different developmental stages}

Tissue distribution assay was conducted with two age classes of abalones. First, from 1-year-old immature juveniles $(21.5 \pm 4.1 \mathrm{~g}$ for total weight; $n=12)$, six kinds of tissues including the gill, gut, heart, hemolymph, hepatopancreas, and muscle (foot muscle) were surgically removed individually. For hemolymph, centrifugation (2500 rpm for $10 \mathrm{~min}$ at $4{ }^{\circ} \mathrm{C}$ ) was carried out in order to collect hemocyte pellet. Second, from 3-year-old sexually mature adults showing a clear sign of ovarian and testicular maturation $(96.4 \pm 13.1 \mathrm{~g}$ for total weight; eight each for female and male), same tissue types abovementioned were obtained and additional ovary and testis were obtained. Upon surgically removed, biological samples were immediately frozen on dry ice and stored at $-80{ }^{\circ} \mathrm{C}$ until used for RNA isolation.

In order to obtain developing embryos and early larvae, artificial insemination of sperm (from three males) to eggs (from eight females) was conducted by using the conventional induced spawning method including an air exposure and ultraviolet-irradiated seawater treatment. Insemination was made with wet-method at $20{ }^{\circ} \mathrm{C}$ and incubated at the same temperature until the end of sampling. An aliquot of developmental samples each consisting of approximately 20,000 30,000 embryos or larvae was sampled at six time points: just before insemination (unfertilized eggs), early cleavages (i.e., $2 \sim 8$ cells stage; at $2 \mathrm{~h}$ post insemination; $2 \mathrm{hpi}$ ), morula (5 hpi), trochophore (12 hpi), early veliger (18 hpi), and late veliger (42 hpi), based on the microscopic examination. Upon sampling, embryos and larvae were also frozen on dry ice and stored at $-80{ }^{\circ} \mathrm{C}$ until used. Two replicate samplings were carried out for each time point.

\section{Experimental in vivo stimulatory challenges}

Two independent stress exposure experiments were carried out: one was with heavy metal exposure and the other with heat shock treatment. For heavy metal exposure, eight juvenile individuals $(24.5 \pm 3.6 \mathrm{~g}$; approximately 1 year old $)$ were assigned into one of five experimental tanks (70-L capacity containing $50 \mathrm{~L}$ of $1-\mu \mathrm{m}$ filtered seawater at $20^{\circ} \mathrm{C}$ ) and acclimated to the tank conditions for $24 \mathrm{~h}$ before heavy metal treatment. The heavy metals used for exposure were of analytical grade reagents (Sigma-Aldrich, St. Louis, MO, USA). After $24 \mathrm{~h}$, two tanks were treated with $0.02 \mathrm{mg} / \mathrm{L}$ (i.e., $20 \mathrm{ppb}$ ) and $0.1 \mathrm{mg} / \mathrm{L}$ (i.e., $100 \mathrm{ppb}$ ) cadmium (Cd), while two tanks with 20 and 100-ppb zinc ( $\mathrm{Zn}$ ) (Lee and Nam 2016a). Nominal concentration of the metal for each metal-exposed group was adjusted by using $\mathrm{CdCl}_{2}$ or $\mathrm{ZnCl}_{2}$ stock solution. Remaining one tank was treated with only 1-mL distilled water that had been used for reconstitution of the metals (i.e., for non-exposed control). For each group, two replicate tanks were prepared identically. Treatment duration was $24 \mathrm{~h}$. At the end of exposure, gill, hemocyte, hepatopancreas, and foot muscle were sampled individually from six randomly chosen individuals as described above. 
On the other hand, for heat shock treatment, 22 individuals ( $21.1 \pm 3.1 \mathrm{~g}$; same-aged as above) were assigned into one of four 100-L tanks (two for heat-stressed groups and two for non-stressed groups) at $20{ }^{\circ} \mathrm{C}$. Each tank was equipped with a custom-designed apparatus for mechanical filtration. After $24 \mathrm{~h}$ of acclimation period, water temperature of the two tanks (heat-stressed groups) was elevated using the adjustable thermostat-assisted aquarium heaters $(400 \mathrm{~W})$ with an increment rate of $1{ }^{\circ} \mathrm{C} / \mathrm{h}$. When the temperature reached $30{ }^{\circ} \mathrm{C}$, the temperature was kept to be constant at $30{ }^{\circ} \mathrm{C}$ for additional $24 \mathrm{~h}$. Samplings were made at $20{ }^{\circ} \mathrm{C}$ (just before thermal elevation), $25{ }^{\circ} \mathrm{C}$ ( $5 \mathrm{~h}$ after elevation started), $30{ }^{\circ} \mathrm{C}(10 \mathrm{~h})$, $30{ }^{\circ} \mathrm{C}+12 \mathrm{~h}\left(12 \mathrm{~h}\right.$ after reaching $30{ }^{\circ} \mathrm{C} ; 22 \mathrm{~h}$ after elevation started), and $30{ }^{\circ} \mathrm{C}+24 \mathrm{~h}\left(24 \mathrm{~h}\right.$ after reaching $30^{\circ} \mathrm{C} ; 34 \mathrm{~h}$ after elevation started). Four individuals were randomly selected from each tank to constitute eight individuals per temperature group at each sampling point. Tissues sampled were gill, hemocyte, hepatopancreas, and foot muscle. Meanwhile, non-stressed control groups were also identically prepared with heat-shock groups, but the temperature $\left(20^{\circ} \mathrm{C}\right)$ was kept to be constant until the end of experiment. At the same sampling point, the identical number of abalones ( $n=4$ per tank) was also obtained from non-stressed control groups. Temperature of each tank was confirmed to be ranged within $\pm 0.5^{\circ} \mathrm{C}$. Dissolved oxygen levels were adjusted to be ranged from 7.5 to $8.5 \mathrm{ppm}$ for all the experimental tanks. Abalones were not fed during stimulatory challenge experiments.

\section{RT-qPCR assay and statistics}

Total RNA was extracted using TriPure ${ }^{\bullet}$ Reagent (Roche Applied Science, Mannheim, Germany) and then purified using RNeasy Mini Plus Kit (Qiagen, Hilden, Germany) including DNase I treatment step. An aliquot $(2 \mu \mathrm{g})$ of total RNA prepared was reverse transcribed into cDNA by using the Omniscript ${ }^{\circ}$ Reverse Transcription Kit (Qiagen, Germany) including oligo-dT primer according to the manufacturer's instruction. Synthesized cDNA was fourfold diluted with sterile distilled water, and an aliquot of $2 \mu \mathrm{L}$ was included in a qPCR reaction as a template. The qPCR reaction was conducted with a LightCycler ${ }^{\circ} 480$ Real-Time PCR System and LightCycler 480 SYBR Green I Master (Roche Applied Science, Germany), according to the manufacturer's instructions. Thermal cycling condition for each gene (i.e., MTF-1 and normalization control genes) can be referred to Additional file 1: Table S1. Based on our preliminary study to evaluate candidate housekeeping genes for the normalization of RT-qPCR amplification (unpublished data; see also (Lee and Nam 2016a; Lee and Nam 2016b)), abalone ribosomal proteins L5 (RPL5; ABO26701) and L7 (RPL7; KP698945) genes were used as reference genes to normalize expression levels of MTF-1 transcripts in tissue samples (i.e., for basal tissue expression assays and stress exposure treatments), while RPL7 and RPL8 (KP698947) were used to normalize MTF-1 expression across developmental samples (embryos and larvae). Additionally, for heavy-metal exposure groups, the messenger RNA (mRNA) expression levels of MTF-1 were compared with those of MT (the known target gene of MTF-1) in order to examine whether or not there might be any positive or proportional relationship in the metalmediated modulation patterns between MTF-1 and MT genes. PCR efficiency of primer pair for each gene was validated to be at least higher than $95 \%$ based on the standard curve prepared using a fivefold serial dilution of cDNA mix. For each cDNA sample, triplicate assays were carried out in an independent fashion.

Quantitative PCR-based MTF-1 mRNA expression levels across tissue types and developmental stages under non-stressed conditions were presented as $\Delta \mathrm{Ct}$ (Ct of the MTF-1 gene subtracted from the Ct of each internal control gene). On the other hand, differential expression levels of metal-exposed or heat shock-treated group relative to their corresponding non-stressed control groups were presented as the fold difference to the non-stressed controls by using the formula $2^{-\Delta \Delta C t}$ (Schmittgen and Livak 2008). Expression levels between or among groups were tested using Student's $t$ test or one-way ANOVA (followed by Duncan's multiple ranged tests). Difference was considered to be significant when $P<0.05$.

\section{Results and discussion}

\section{Characteristics of abalone MTF-1 CDNA and deduced} amino acid sequences

The full-length cDNA of abalone MTF-1 was comprised of 48-bp 5'-untranslated region (UTR), a 1509-bp single open reading frame (ORF) encoding a polypeptide of 503 amino acids, and 582-bp 3'-UTR including a stop codon and 19-bp poly $(\mathrm{A}+)$ tail. A putative polyadenylation signal (AATAAA) was found at $21 \mathrm{bp}$ prior to the poly $(\mathrm{A}+$ ) tail (GenBank accession number; KT895224) (Additional file 1: Figure S1). The MTF-1 protein based on the deduced amino acid sequence was estimated to have $54.86 \mathrm{kDa}$ of calculated molecular mass and 5.51 of theoretical pI value, respectively. Abalone MTF-1 represented quite a low sequence homology to its vertebrate and invertebrate orthologs where the maximum sequence identity at amino acid level was found to be only $27 \%$ with Biomphalaria glabrata (air-breathing freshwater snail; Gastropoda; Mollusca). Non-conservative feature without any appreciable sequence similarity to other MTF-1s is also found in the putative activation. Abalone MTF-1 was likely to possess only a shortened fragment (44-aa; $\mathrm{pI}=4.06)$ presumed for the acidic domain and to lack almost entire region corresponding to the proline-rich domain and serine/threonine-rich 
domain of vertebrate MTF-1 orthologs (Additional file 1: Figure S2).

In contrast, the abalone MTF-1 was proven to share a high structural homology with other MTF-1s in the DNA binding domain (Fig. 1). All the MTF-1 proteins including the abalone MTF-1 (but except for the shortest Octopus bimaculoides MTF-1 having only five zinc fingers) were found to show highly conserved six $\mathrm{C}_{2} \mathrm{H}_{2}$-zinc fingers in their DNA binding domains. In their DNA binding domains, 12 cysteine residues and histidine residues were clearly conserved, in which the zinc finger domain of abalone MTF-1 showed the highest sequence identity (77\%) with that of orthologue isoforms from Crassostrea gigas (Bivalve, Mollusca). However, the molecular phylogenetic analysis using the DNA binding domains has indicated that MTF-1s have been largely divergent in the molluscan phylum, which is apparently different form the monophyletic clustering of orthologs from Chordata phylum (Additional file 1: Figure S3). Functional partition of the zinc finger domains in abalone MTF-1 has been remained to be further characterized; however, from the mammalian studies, the region from the second to fourth zinc fingers have been proposed to constitute the core DNA binding domain while the first finger has been reported to serve as a metal-sensing domain (Bittel et al. 2000). Within each zinc finger, the His-X-Arg/Lys-X-His [H-X-(R/K)-X-H where $\mathrm{X}$ is any amino acid] motif has been known as a key site for zinc binding (Günther et al. 2012a), and it is preserved in the 1st to 5 th zinc fingers for all the species examined in the present study. However, in the last finger

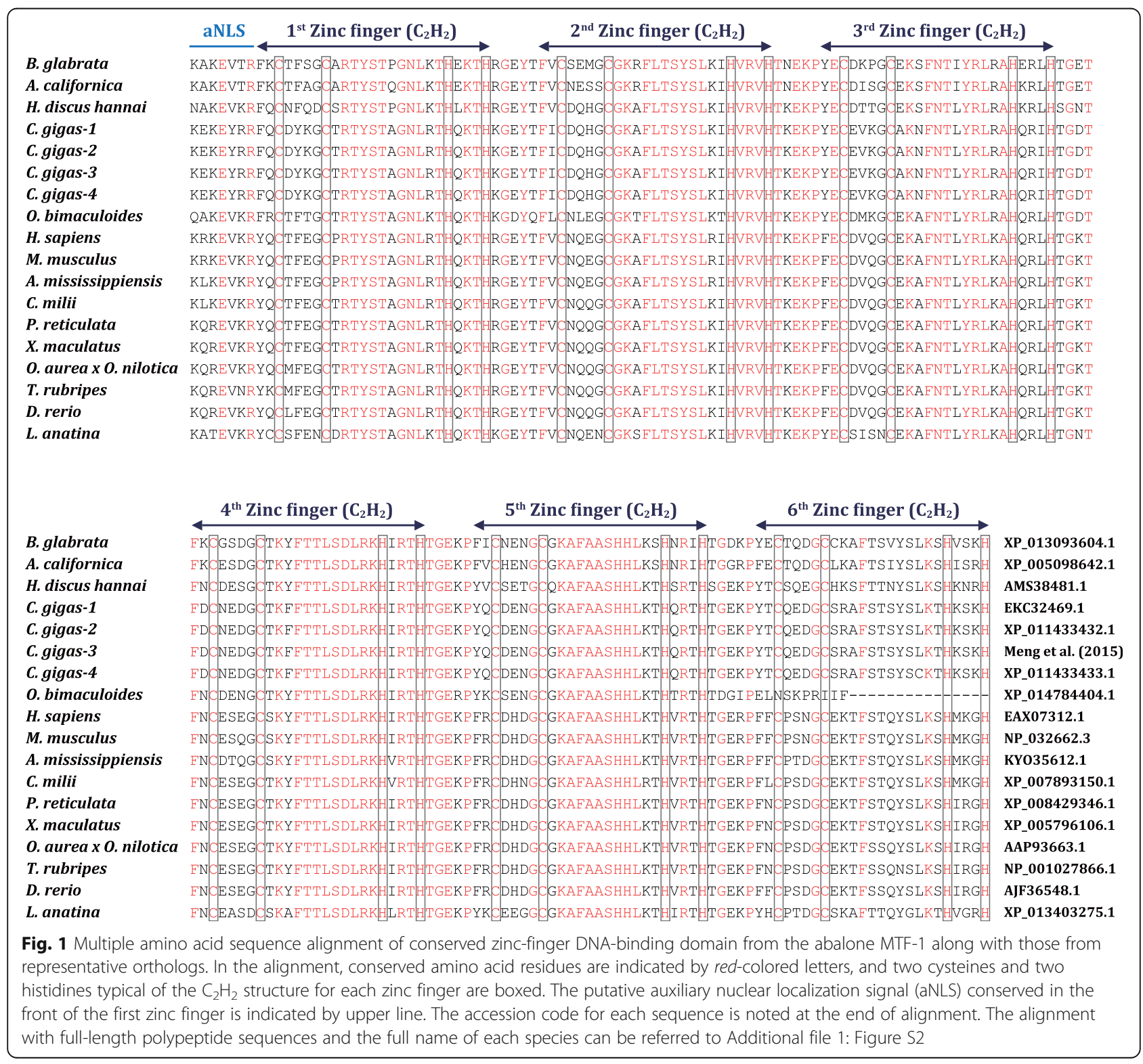


(i.e., the 6th finger), the Arg/Lys residue inside the 5amino-acid stretch was found to be conserved only in vertebrate MTF-1s. Meanwhile, all the molluscan MTF-1s showed a phenylalanine (Phe) as the first amino acid of the first zinc finger, whereas vertebrate and brachiopod orthologs possessed a tyrosine (Tyr) (Cheung et al. 2010).

Abalone MTF-1 showed both conserved and unique features in the peptide linkers connecting zinc fingers. Conserved peptide linkers were found between 1st and 2nd fingers (Arg-Gly-Glu-Tyr-Thr), between 2nd and 3rd fingers (Thr-Lys-Glu-Lys-Pro), and between 4th and 5th fingers (Thr-Gly-Glu-Lys-Pro). On the other hand, unique linkers were found between 3rd and 4th fingers [Ser-Gly-Asn-Thr in abalone vs. Thr-Gly-Lys-Thr in vertebrates vs. Thr-Gly-(Glu/Asn/Asp)-Thr in other molluscs and a brachiopod species] and between 5th and 6th fingers [Ser-Gly-Glu-Lys-Pro in abalone vs. Thr-GlyGlu-(Lys/Arg)-Pro in vertebrates vs. Thr-Gly-(Asp/Gly/ Glu)-(Lys/Arg)-Pro in others]. Peptide linkers in multizinc finger domains have been reported to play roles in not only structural stabilization but also interfinger interactions for DNA-binding affinity of the zinc finger domains (Li et al. 2006). Particularly in the MTF-1, the linker between 1st and 2nd fingers has been known to be crucial in the zinc-sensing ability of the MTF-1 with regard to the formation of the ternary (MTF-1-zincDNA) complex for activating MT gene transcription (Li et al. 2006). Taking this into account, the abalone MTF-1 is thought to preserve a fundamental property of zinc-sensing function similarly with mammalian orthologs since it conserves a completely identical linker (i.e., the Arg-Gly-Glu-Tyr-Thr linker between 1st and 2nd fingers) with mammalian MTF-1s. However, the present abalone MTF-1 showed apparent dissimilarity with vertebrate orthologs in the linkers between 3rd and 4th fingers and between 5th and 6th fingers. Particularly because the linker between 3rd and 4th fingers has been reported to be important for the in vivo and in vitro sensitivity of zinc-dependent activation of MTF-1 (Li et al. 2006), this linker could be a good target for future studies to examine the potential difference in the linker-mediated zinc-finger function between abalone MTF-1 and mammalian/vertebrate orthologs.

In the abalone MTF-1, a putative auxiliary nuclear localization signal (aNLS) was identifiable in the front of the first zinc finger, as similarly with all other MTF-1 orthologs. Of seven amino acid residues to comprise the auxiliary NLS, the positions of two amino acids $\left({ }^{3} \mathrm{Glu}\right.$ and ${ }^{7} \mathrm{Arg}$ ) are conserved in all the MTF-1s examined (Fig. 1). However, due to the potential deletion in the putative acidic domain, no canonical nuclear export signal (NES) was observable in the abalone MTF-1 unlike vertebrate orthologs. Within a concept of MTF-1 activation (i.e., a nuclear-cytoplasmic shuttling function established in mammalian MTF-1), NLS and NES are responsible for balanced subcellular distributions of MTF-1 proteins (i.e., import and export, respectively) under both stressed and non-stressed conditions (Günther et al. 2012a). The NES motif in vertebrate MTF-1 is usually embedded in the acidic activation domain (Günther et al. 2012a; Cheung et al. 2010). However, the abalone MTF-1 does not show any typical NES motif, although a putative NLS motif is predicted in the front of the first zinc-finger domain that are also conserved all MTF-1s examined (i.e., conserved auxiliary NLS). The absence of acidic domain-embedded NES is not limited to abalone MTF-1, i.e., all molluscan MTF$1 \mathrm{~s}$ are also likely to lack the NES at the corresponding region. Hence, our finding may suggest that molluscan MTF-1s could be different from mammalian MTF-1s in their subcellular localization control under both basal and stimulated conditions. A recent study with an oyster species (C. gigas) has claimed that the MTF-1 would primarily localize in the nucleus even under unstressed conditions and nuclear translocation might be uncritical for the activation of the oyster MTF-1 (Meng et al. 2015).

In addition, the typical motif of cysteine cluster (consensus sequence $=$ Cys-Gln-Cys-Gln-Cys-Ala-Cys) that could be commonly found in the region immediately following the serine/threonine-rich domain of vertebrate MTF-1s was not detected in the abalone MTF-1 (Additional file 1: Figure S2). The cysteine cluster has been reported to be essentially necessary for metalinduced transcriptional activity and homodimerization of mammalian MTF-1 (Günther et al. 2012a; Günther et al. 2012b). Like with NES abovementioned, none of molluscan MTF-1 represents a canonical C-terminus cysteine cluster, suggesting that molluscan MTF-1s might have different mechanism(s) for metal-induced transcription (i.e., recruitments of transcriptional cofactor partners in the promoter/enhancer of target genes).

\section{Prediction of transcription factor binding sites}

From the bioinformatic prediction, the 1691-bp 5'-upstream region from the ATG translation start site of the abalone MTF-1 gene represented various transcription factor binding sites (Fig. 2) (Additional file 1: Figure S4). The abalone MTF-1 promoter revealed a canonical TATA box (TATAAA) at -474 bp (from the ATG). Importantly, it represented a copy of MRE (TGCRCNC; -959 bp), suggesting the possible modulation of MTF-1 itself by heavy metal-driven cellular stressors, which is clearly inconsistent with the lack of MRE in many mammalian MTF-1 promoters (Auf der Maur et al. 2000; Bi et al. 2006). In addition, a xenobiotic response element (XRE; TNGCGTG; $-736 \mathrm{bp}$ ) was predicted in the abalone MTF-1 promoter. XRE is an aryl hydrocarbon receptor (AhR)-targeted motif involved in the ligand 


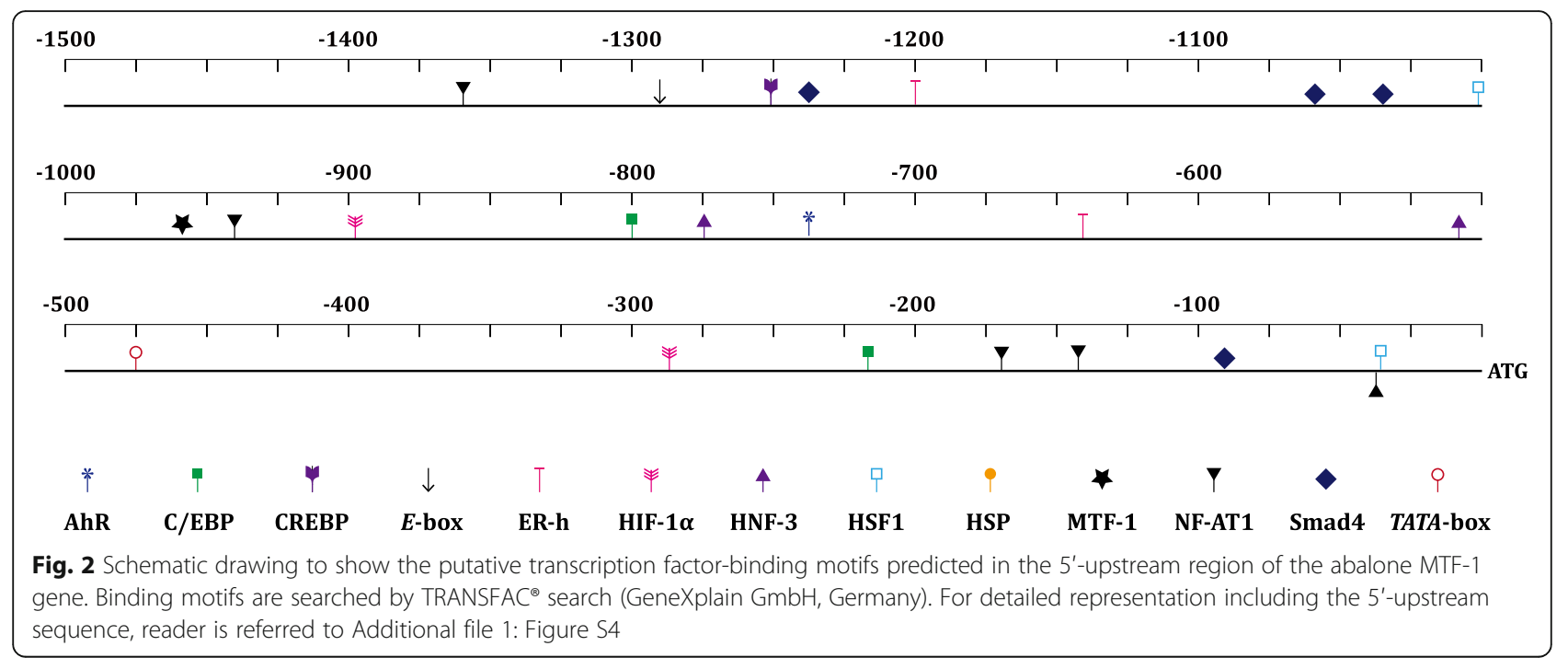

(i.e., 2,3,7,8-tetrachlorodibenzodioxin (TCDD))-activated pathway to detoxify the effects of TCDD-related compounds. However, because invertebrate AhR homologues have been reported to lack the ability to bind TCDD directly (Hahn et al. 2006), the molecular mechanism on the potential interconnection between MRE/MTF-1 and $\mathrm{XRE} / \mathrm{AhR}$ paths should be further explored. Nevertheless, recent mammalian studies have also highlighted multitasking roles of AhR in various signaling pathways associated with cell cycle control and antioxidant protection against oxidative stresses (Jackson et al. 2015). Besides, several transcription factor binding sites such as heat shock element (HSE; GAANRTTC; -1003 and -32 bp; targeted by heat shock factor (HSF)), hypoxia response element (HRE; RCGTG; -895 and -285 bp; by hypoxiainducible factor-1 alpha (HIF- $1 \alpha)$ ), and other sites recognized by cyclic AMP response element binding protein (CREBP; TGACGY; -1253 bp) and nuclear factor for activated T-cells (NF-AT1; WGGAAA; -941, -169, and $-33 \mathrm{bp}$ ) were predicted. All of these factors have been known to be related with stress responses of animals (Saydam et al. 2003; Dubé et al. 2011). Abalone MTF-1 promoter also revealed motifs that might be targeted by transcription factors generally known to be involved in development, signal transduction, cell proliferation and/or organ development. They included Smad4, hepatocyte nuclear factor (HNF), CCAAT-enhancer binding protein $(\mathrm{C} /$ EBP), E-box binding protein, and estrogen receptor (ER).

\section{Tissue distribution and basal expression levels}

Based on the RT-qPCR analysis with immature juvenile abalones, MTF-1 mRNAs were detected in all the tissue types examined; however, basal expression levels were quite variable among tissues (Fig. 3). The ubiquitous detection of MTF-1 transcripts across all the tissues is not surprising when taken into account its housekeeping and fundamental roles in most cell types (Auf der Maur et al. 2000; O'Shields et al. 2014). The MTF-1 mRNAs were robustly expressed in hemocytes $(P<0.05)$, and this highest expression level was followed by muscles and gills, whereas the least mRNA expression was found in heart $(P<0.05)$. However, this expression pattern was not fully reproducible when measured with the sexually mature adults, although the broad pattern was consistent with findings from juveniles. Unlike in juveniles, the expression level of MTF-1 in the gut was found to be as high as that in the hemocytes in both female and male adults. More noticeably, matured adult abalones displayed a strikingly apparent difference in the MTF-1 expression in gonads where the extraordinarily high expression level was observed in testis while only minute expression in ovary (more than 150-fold difference; $P<0.05)$. Testis-predominant expression pattern of abalone MTF-1 in this study is similar with previous findings made in mouse (Auf der Maur et al. 2000) and hybrid tilapia (Oreochromis aurea $\times$ Oreochromis nilotica) (Cheung et al. 2010), collectively suggesting the possible involvement of MTF-1 in the male reproduction. Yet, the mechanism underlying the robust expression of MTF-1 in the abalone testes is currently unknown and open to hypothesize. In mouse, the reason for the high MTF-1 expression in testis has been explained by that sexually mature mice need to accumulate a large quantity of MTs in their testes (Auf der Maur et al. 2000; De et al. 1991). However, this hypothesis is unlikely to be adopted to this abalone species since the virtual increase of MT expression in sexually mature abalones has been observed in ovary rather than in testis (Lee and Nam 2016a), suggesting the molecular mechanism for the boosted expression of MTF-1 in abalone testis might be distinct from the ones in mammals. Hence, further study to monitor the MTF-1 expression in line with the testis development and 

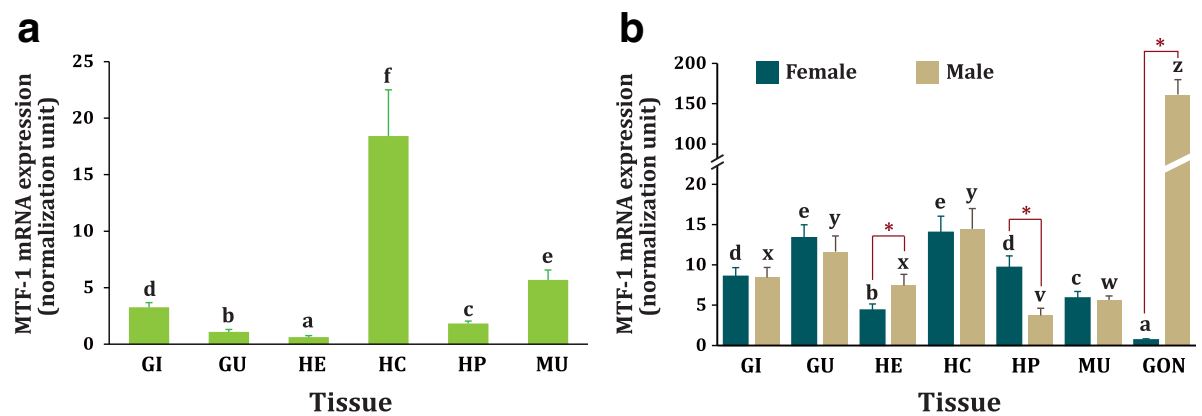

Fig. 3 Tissue distribution patterns and basal expression levels of abalone MTF-1 mRNAs in a immature juvenile and b sexually matured adult tissues, based on RT-qPCR assays using the normalization against RPL5 and PPL7 references. Tissue abbreviations are gill (G), gut (GU), heart (HE), hemocyte $(H C)$, hepatopancreas $(H P)$, muscle $(M U)$, and gonad (GON; i.e., ovary or testis). In a, histograms with different letters indicate the significantly different means based on ANOVA followed by Duncan's multiple ranged tests at $P<0.05$. In $\mathbf{b}$, the significantly different means within a sex are indicated by $a-e$ (for females) and $v-z$ (for males) base on ANOVA followed by Duncan's multiple ranged tests at $P<0.05$. The significantly different means between sexes observed in heat, hepatopancreas, and gonad tissues are noted by asterisks based on Student's $t$ test analysis at $P<0.05$

maturation cycle would be valuable to get a deeper insight into this phenomenon. Besides the gonadal expression, matured abalones showed a sex-specific difference in the basal expression of MTF-1 in heart and hepatopancreas. Male abalones displayed higher expression in heart than females did whereas female abalones showed higher expression in hepatopancreas than males did. This finding is similar with a previous observation with zebrafish where males should have a higher MTF-1 mRNA expression in the heart than females (O'Shields et al. 2014). Although it has been still inconclusive for such a sex-related dimorphism, the response of zebrafish MTF-1 to Cd exposure has been reported to be gender dependent in some tissues (O'Shields et al. 2014).

\section{Developmental expression}

The MTF-1 mRNAs were found to be already present in unfertilized eggs based on RT-qPCR assay, which could be considered as a typical indicative sign of the maternal contribution of MTF-1 to offspring. Fine regulation of metal homeostasis should be one of the prerequisite requirements for developmental success of marine mollusc embryos that undergo external development in metalresiding sea water (Roesijadi et al. 1996; Jenny et al. 2006; Mao et al. 2012). The initial expression level was decreased down in early cleavage stages and rebounded to the initial level at morula stage. Although there was a trend toward increase of MTF-1 mRNAs with the developmental progress up to early veliger stage, the degree of upregulation was only modest. When the development progressed to late veliger stage, the mRNA expression level of MTF-1 was significantly elevated $(P<0.05)$ (Fig. 4). Developmental expression of MTF-1 in marine molluscan animals has not been yet characterized previously. However, the expression pattern observed in this study was generally in congruent with the anticipated roles of MTF-1 in embryonic and early ontogenic developments, as inferred from mammalian and teleostean cases (Günes et al. 1998; Chen et al. 2002; Chen et al. 2007). The expression pattern of MTF-1 during the development was also in agreement with the modulation pattern of its primary target (e.g., MT) in the same abalone species (Lee and Nam 2016a). Previously, the functional involvements of MTF-1 in the development and organogenesis have been highlighted by the lethality of "MTF-1-knockout" mice (Günes et al. 1998; Wang et al. 2004) and by induced inhibition of MTF-1 signaling followed by transcriptomic profiling in zebrafish embryos (O'Shields et al. 2014).

\section{Expression during $\mathrm{Zn}$ and $\mathrm{Cd}$ exposure}

There was an apparent difference between the two metal ions in the modulation of MTF-1 gene expression. In overall, $\mathrm{Cd}$ induced potently the mRNA expression of

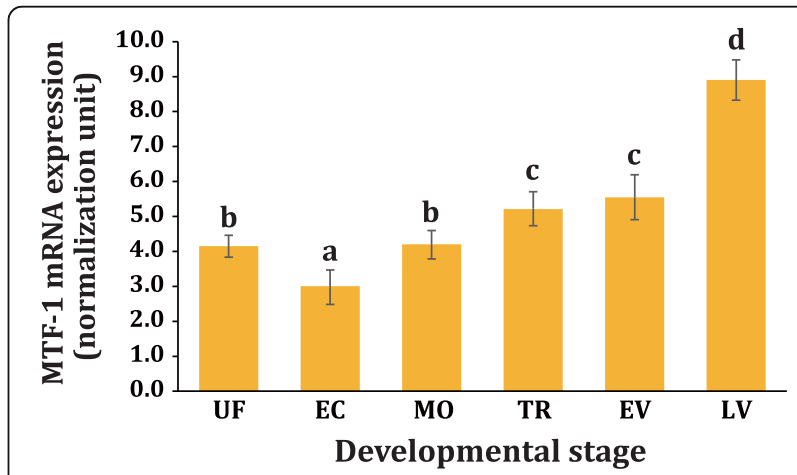

Fig. 4 Expression of abalone MTF-1 in developing embryos and early larvae assessed by RT-qPCR analysis using RPL7 and PPL8 reference genes as normalization controls. Abbreviations for developmental samples are unfertilized eggs (UF), eggs at early cleavages (EC; 2 8 cells stage), eggs at morula $(M O)$, trochophore (TR), early veliger (EV), and late veliger ( $L V$. Histograms with different letters indicate the significantly different means based on ANOVA followed by Duncan's multiple ranged tests at $P<0.05$ 
MTF-1 while on the contrary, Zn repressed the MTF-1 expression (Fig. 5). Differential expression patterns of MTF-1 in response to $\mathrm{Zn}$ and $\mathrm{Cd}$ (20 and $100 \mathrm{ppb}$ for both metals) were also dependent upon tissue types examined (gill, hepatopancreas, muscle, and hemocyte). In the gill, the exposure with 20-ppb $\mathrm{Cd}$ strongly induced the MTF-1 $(P<0.05)$ but higher exposure dose $(100 \mathrm{ppb})$ did not give rise to the significant modulation of MTF-1 $(P>0.05)$. However, exposure with both doses of Zn significantly downregulated the MTF-1 in the gill $(P<0.05)$. On the other hand, in the hepatopancreas, both doses of Cd elevated the MTF-1 mRNA levels in a dose-dependent fashion $(P<0.05)$. Although the $20-\mathrm{ppb}$ $\mathrm{Zn}$ exposure exhibited the small increase of MTF-1 mRNA levels in the hepatopancreas, 100-ppb Zn did not show any significant difference as compared to the level observed in non-exposed control $(P>0.05)$. Unlike in other three tissues, $\mathrm{Cd}$ exposure was unable to induce the MTF-1 expression in muscle tissue. In the muscle, $\mathrm{Zn}$ exposure depressed the MTF-1 mRNA expression (more significant downregulation of MTF-1 in the $100-\mathrm{ppb}$ exposed group than in the 20-ppb exposed group). For hemocytes, the group exposed with 100-ppb $\mathrm{Cd}$ displayed a small, but statistically significant, increase of MTF-1 expression, while again, Zn-exposure resulted in the rapid downregulation of MTF-1 irrespective of exposure doses $(P<0.05)$.

In contrast to the variable or opposite regulation of MTF- 1 by $\mathrm{Cd}$ and $\mathrm{Zn}$, the transcriptional response of MT (the main target of MTF-1) to the metal exposure treatments was relatively uniform. Further, unlike MTF-1 showing the downregulation upon $\mathrm{Zn}$ exposure in most instances, MT was found to be consistently upregulated by $\mathrm{Zn}$ in both 20 and 100-ppb exposure treatments $(P<0.05)$. Although the induced folds were variable among tissues, MT gene expression was unfailingly induced in all the four tissues by exposure treatments with 100-ppb Cd, 20-ppb Zn, and 100-ppb Zn, but not by 20ppb Cd. Collectively, the modulation patterns upon metal exposure were apparently different between MTF-1 and MT genes, and the degree of $\mathrm{Cd}$-mediated upregulation in each tissue was much higher for MT than MTF-1 (Fig. 5).

Previous studies have indicated that mammalian MTF1 should be a constitutively expressed gene with a TATA-less promoter and that mammalian MTF-1s would not show any appreciable response to experimentally designed heavy metal and other stress factors. The plausible reason for the absence of metal (or stress) responsiveness has been explained by the lack of MRE motif in the mammalian (e.g., mouse and human) MTF-1 gene promoters (Auf der Maur et al. 2000; Bi et al. 2006). Hence, the MTF-1 activity in mammals is widely agreed to be largely induced at a post-translation level (Günther et al. 2012a; Smirnova et al. 2000; Saydam et al. 2001). However, in the present study, this abalone MTF-1 gene was proven to possess a putative MRE copy as well as a canonical TATA box in its promoter region. Accordingly, the present abalone MTF-1 gene displayed the Cdmediated induction in multiple tissues (in gill, hepatopancreas and hemocytes, but not in muscles). Similarly, the Cd-induced expression of MTF-1 gene has also been reported in other cold-blooded animals such as zebrafish
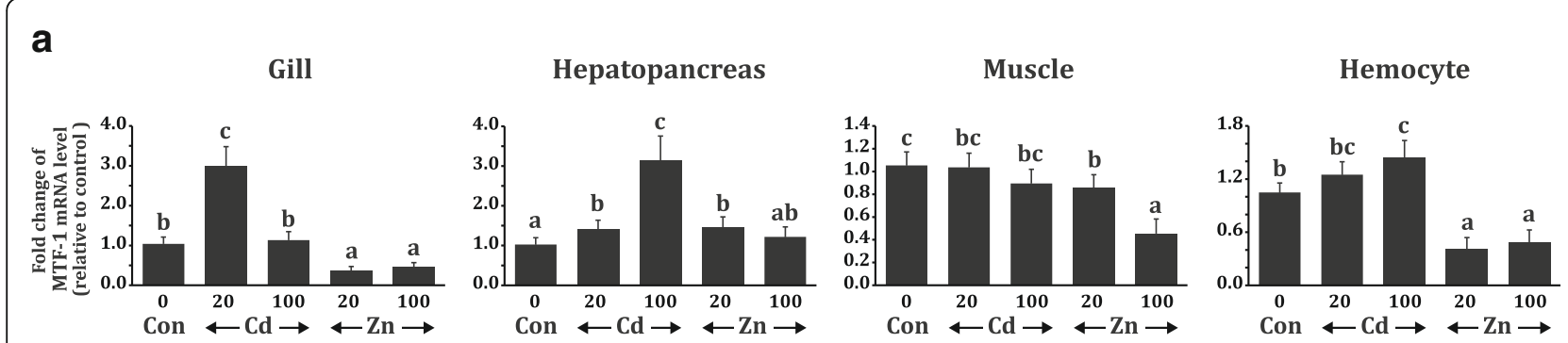

b
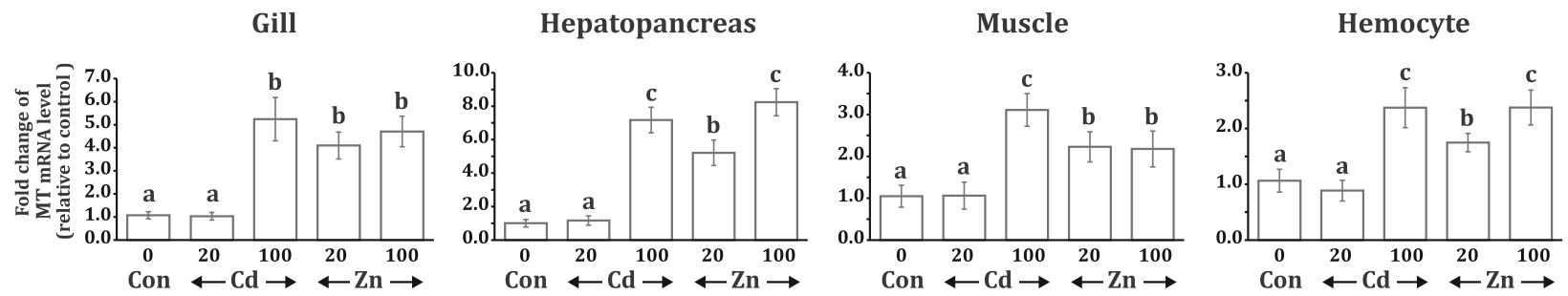

Fig. 5 Transcriptional responses of abalone MTF-1 (a) and MT (b) to experimental heavy metal exposures. Metal exposures were done with cadmium or zinc with the dose strengths of 20 and $100 \mathrm{ppb}$ for $24 \mathrm{~h}$. RT-qPCR data were presented as fold changes of MTF-1 mRNAs in expressed groups relative to that of non-exposed control (Con) based on the normalization against two internal control genes (RPL5 and PPL7). Histograms with different letters indicate the significantly different means based on ANOVA followed by Duncan's multiple ranged tests at $P<0.05$ 
(Dubé et al. 2011; Cheuk et al. 2008) and carp (Ferencz and Hermesz 2009), which is obviously different from the principle for the MTF-1 regulation in mammals (Bi et al. 2006; Saydam et al. 2001).

However, the transcription of abalone MTF-1 was not induced by zinc except only a minute increase in the hepatopancreas; furthermore, $\mathrm{Zn}$ exposure even downregulated the mRNA expression of abalone MTF-1 in other tissues. This finding is similar with no induction of MTF-1 mRNA observed for zebrafish cells exposed to $\mathrm{Zn}$ (Cheuk et al. 2008), although $\mathrm{Zn}$ exposure in zebrafish was reported to be able to activate the nuclear translocation of MTF-1 from cytoplasm (Chen et al. 2007). On the other hand, a recent study with oyster $C$. gigas has shown that mRNA expression level of MTF-1 could be increased by $\mathrm{Zn}$ exposure, but the induced amount was only modest even treated with high dose of $\mathrm{Zn}$ (Meng et al. 2015). In the same report, authors have indicated that RNA interference of MTF-1 would result in the depression of Zn-mediated induction of MT (the known target of MTF-1), consequently confirming that MTF-1 is a superordinate regulator of the MT (Meng et al. 2015). However, in this study, the inducible pattern of MTF-1 was not in agreement with that of MT expression. Both Cd (inducer of abalone MTF-1) and Zn (non-inducer of abalone MTF-1) were found to be able to induce MT expression in all the tissues examined. Further, the quantitative relationship between MTF-1 and MT expression was not proportional. Currently, it has been widely agreed that $\mathrm{Zn}$-mediated induction of MT could be achieved by direct binding of $\mathrm{Zn}$ to the MTF-1 fingers, and other metals such as $\mathrm{Cd}$ and $\mathrm{Cu}$ may not replace $\mathrm{Zn}$ in zinc finger binding (Schmittgen and Livak 2008; Chen et al. 1999; Zhang et al. 2001). Based on this, the Cd-mediated induction of MT gene might be considered as an indirect consequence. Possibly, Cd load might give rise to the release of $\mathrm{Zn}$ from MTs (also from other metalloproteins), and the increase of cellular Zn levels may activate MTF-1 through the Zn-binding. This zinc pool hypothesis can also be applied to the MT induction upon exposed to diverse oxidative stresses (Günther et al. 2012a). Hence, the controversy between MTF-1 and MT responses to zinc should be challenged in future study by examining cellular $\mathrm{Zn}$ concentrations (or tissue burden) under various Zn-exposure conditions.

\section{Response to heat shock treatment}

Abalone MTF-1 was proven an early phase, heat-shock responsive protein as evidenced by the rapid modulation upon thermal increases. The MTF-1 mRNA levels in the gill were rapidly increased (fourfold relative to $20{ }^{\circ} \mathrm{C}$; $P<0.05)$ as early as when water temperature reached $25{ }^{\circ} \mathrm{C}$ (Fig. 6). The expression level was further elevated when the temperature reached $30{ }^{\circ} \mathrm{C}$ (i.e., designated $30{ }^{\circ} \mathrm{C}+0$ h) $(P<0.05)$. Afterward, the MTF-1 expression levels began to be decreased down with the continued incubation at $30{ }^{\circ} \mathrm{C}$ (i.e., $30{ }^{\circ} \mathrm{C}+12 \mathrm{~h}$ and $30{ }^{\circ} \mathrm{C}+24 \mathrm{~h}$ ); however, the expression level at the end of thermal treatment was still significantly higher than that of $20^{\circ} \mathrm{C}$ group $(P<0.05)$. On the other hand, in the hepatopancreas, the beginning of MTF-1 upregulation was more or less lagged as compared to that in the gill. Significant induction of MTF-1 in the hepatopancreas became evident from the $30{ }^{\circ} \mathrm{C}+12 \mathrm{~h}$ group, and the elevated expression level remained constant at $30{ }^{\circ} \mathrm{C}+24 \mathrm{~h}(P<0.05)$. In muscles, the significant induction of MTF-1 was found at $30{ }^{\circ} \mathrm{C}+0 \mathrm{~h}$ and $30{ }^{\circ} \mathrm{C}+12 \mathrm{~h}$ but soon returned to the initial level observed at $20{ }^{\circ} \mathrm{C}$ group. Meanwhile, hemocyte displayed the rapid induction of MTF- 1 mRNA at $25{ }^{\circ} \mathrm{C}$; however, the expression dropped sharply at $30{ }^{\circ} \mathrm{C}+0 \mathrm{~h}$ in which the decreased expression level was even lower than that of $20{ }^{\circ} \mathrm{C}$ group $(P<0.05)$. Afterward, the MTF-1 expression rebounded at $30{ }^{\circ} \mathrm{C}+0 \mathrm{~h}$ group. Then, the rebound was followed by a further increase at $30{ }^{\circ} \mathrm{C}+12 \mathrm{~h}$ group $(P<0.05)$ (Fig. 6).

As aforementioned, the zinc pool sensing mechanism by MTF-1 upon exposure to stress factors might be adopted for the involvement of MTF-1 in host defense pathways against oxidative stress, since the abrupt and substantial changes of water temperature might be a causative factor to generate oxidative stress in poikilothermal invertebrates (Kim et al. 2007; Attig et al. 2014; Banni et al. 2014). Accordant with this explanation, the potential involvement of MT protein in the heat shock response has already been reported in this abalone species and also in other aquatic animals (Lee and Nam 2016a; Negri et al. 2013; Jarque et al. 2014). Within this context, the cold shock-induced MTF-1 has been reported in the common carp (Cyprinus carpio) brain, with an explanation that sudden temperature drop gave rise to the alteration of physiologically accessible $\mathrm{Zn}$ concentrations in that tissue (Ferencz and Hermesz 2008).

Meanwhile, a series of mammalian studies has proposed that MT and heat shock protein (HSP) genes might work in a non-cooperative way in their transcriptional responses to stress treatments. Experimental evidences for this proposal may include that (1) HSF should boost the activity of HSP gene promoter but hardly affect an MRE-containing promoter of MT gene upon heat shock and metal exposure, (2) heat shockinduced nuclear translocation of MTF-1 has been shown to be insufficient to activate a MT gene promoter, (3) diverse target gene searches for MTF-1 have been indicative of HSP genes as non-affected genes, and (4) MTF-1 has been likely to repress HSF-regulated genes through a direct protein-protein interaction (Lichtlen et al. 2001; Saydam et al. 2003; Uenishi et al. 2006). However, on the 

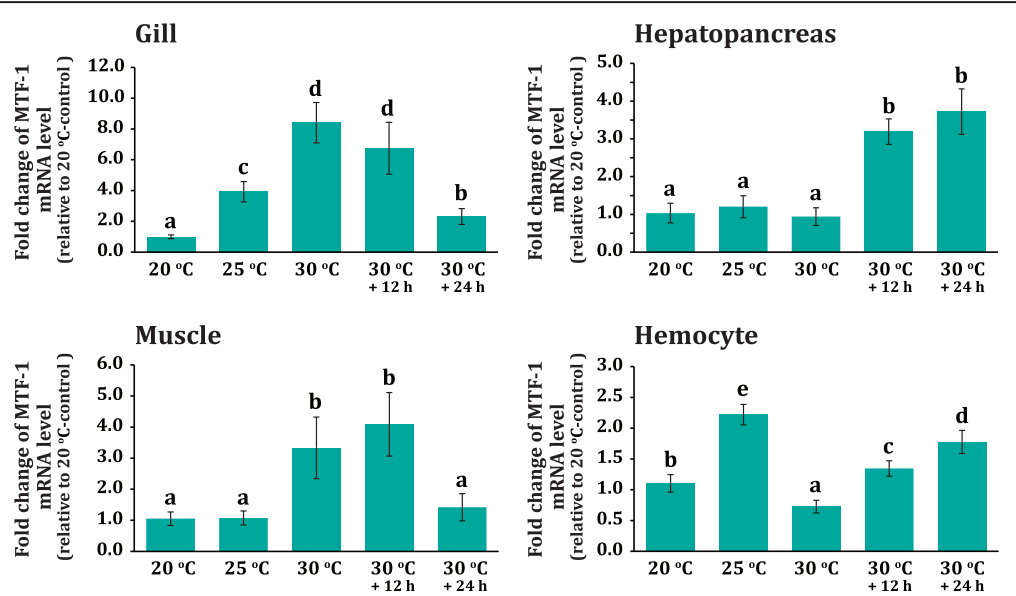

Fig. 6 Differential mRNA expression patterns of abalone MTF-1 in response to heat shock treatments. During heat elevation with an increase rate of $1{ }^{\circ} \mathrm{C} / \mathrm{h}$, abalones were sampled at $20^{\circ} \mathrm{C}$ (just before the elevation), $25^{\circ} \mathrm{C}$, and $30{ }^{\circ} \mathrm{C}$. After reaching $30{ }^{\circ} \mathrm{C}$, abalones were exposed at the constant $30^{\circ} \mathrm{C}$ and sampled after $12 \mathrm{~h}\left(30^{\circ} \mathrm{C}+12 \mathrm{~h}\right)$ and $24 \mathrm{~h}\left(30^{\circ} \mathrm{C}+24 \mathrm{~h}\right)$, respectively. RT-qPCR data were presented as fold changes of MTF-1 mRNAs in expressed groups relative to that of non-heat shocked group (i.e., $20^{\circ} \mathrm{C}$ control) based on the normalization with $R P L 5$ and $P P L 7$ reference genes. Histograms with different letters indicate the significantly different means based on ANOVA followed by Duncan's multiple ranged tests at $P<0.05$

contrary, the present study has shown that the transcription of abalone MTF-1 could be directly activated by heat shock. Hence, our finding suggests that the thermal stress-mediated activation of MTF-1 in abalone may be achieved not only at a post-translation level (i.e., indirectly based on cellular zinc sensing abovementioned) but also at a transcriptional level (i.e., de novo synthesis). The presence of potential HRE motifs in the abalone MTF-1 gene regulatory region is also in congruent with the present hypothesis. Possibly, the activation of MTF-1 from the dual routes is likely to be advantageous for permitting the poikilothermal animals to prepare more efficiently antioxidant components (i.e., antioxidant enzyme genes containing MREs in their promoters as well as MT) against the oxidative stress caused by thermal fluctuations (Kim et al. 2007; Cho et al. 2009). However, further efforts are needed to get direct evidence on the cooperativity between HSF and MTF-1 in this abalone species.

\section{Conclusions}

Novel MTF-1 was isolated and characterized from a commercially important marine mollusc species, Pacific abalone ( $H$. discus hannai). The abalone MTF-1 was found to share a conserved feature in zinc finger, DNAbinding domain with its orthologs; however, it represented non-conservative features in remaining other parts. Bioinformatic analysis of the $5^{\prime}$-upstream region has predicted diverse transcription factor binding motifs that are potentially related with metal regulation, stress responses, and development. Abalone MTF-1 was ubiquitously detected in various tissues, in which the highest expression level was observed in the testes of mature males. Abalone MTF-1 was expressed during the entire period of embryonic and early ontogenic development. From the heavy metal exposure, abalone MTF-1 was found to be $\mathrm{Cd}$ inducible (but not by $\mathrm{Zn}$ ); however, the induced amounts were only modest as compared to that of MT. Abalone MTF-1 was highly modulated in responsive to heat shock potentially via both indirect zinc pool sensing and direct de novo transcription. Data from this study could be a useful basis to approach various researches regarding the stress responses in this abalone species particularly including detoxification of heavy metals and adaptation to thermal stresses.

\section{Additional file}

Additional file 1: Table S1. Summarized information on oligonucleotide primers used in this study. Figure S1. Full-length cDNA and deduced amino acid sequences of abalone Haliotis discus hannai metal responsive transcription factor-1 (MTF-1). In the nucleotide sequence, stop codon is indicated by an asterisk and putative polyadenylation signal is underlined. On the other hand, in the amino acid sequence (in a singlet code), presumed nuclear localization signal (NLS) is underlined (in the front of the first zinc finger), while six putative $\mathrm{C}_{2} \mathrm{H}_{2}$-zinc fingers in the DNA-binding domain are boxed. Figure S2. Multiple sequence alignments of abalone MTF-1 along with its representative orthologs. Putative NLS, six $\mathrm{C}_{2} \mathrm{H}_{2}$-zinc fingers, NES embedded in transactivation domain, and C-terminal Cys-cluster are indicated. Figure S3. Neighbor-joining phylogenetic tree based on amino acid sequences of zin-finger DNA-binding domain and NLS region in MTF-1 orthologs. Tree was computed by poisson correction method using MEGA 7.0 program. Confidence level of each clade was evaluated with bootstrap testing (1000 replicates). Figure S4. Putative transcription factor-binding motifs predicted in the $5^{\prime}$-flanking upstream region of the abalone MTF-1 gene. Transcription factor sites were predicted with perfect and imperfect matches to consensus core sequences. TRANSFAC ${ }^{\circ}$ search (GeneXplain $\mathrm{GmbH}$, Germany) was carried out with the cut-offs scores $>0.99$ (core score) and $>0.95$ (matrix score).

Acknowledgements Not applicable. 


\section{Funding}

This study was supported by the grant from the Golden Seed Project (GSP), Ministry of Oceans and Fisheries, Republic of Korea.

\section{Availability of data and materials}

Not applicable.

\section{Authors' contributions}

SYL carried out molecular cloning, bioinformatics, and expression analyses. YKN designed this study, evaluated data, and drafted the manuscript. Both authors read and approved the final manuscript.

\section{Competing interests}

The authors declare that they have no competing interests.

\section{Consent for publication}

Not applicable.

\section{Ethics approval and consent to participate}

Not applicable.

\section{Publisher's Note}

Springer Nature remains neutral with regard to jurisdictional claims in published maps and institutional affiliations.

Received: 23 January 2017 Accepted: 5 June 2017

Published online: 03 July 2017

\section{References}

Attig H, Kamel N, Sforzini S, Dagnino A, Jamel J, Boussetta H, Viarengo A, Banni M. Effects of thermal stress and nickel exposure on biomarkers responses in Mytilus galloprovincialis (Lam). Mar Environ Res. 2014;94:65-71.

Auf der Maur A, Belser T, Wang Y, Günes C, Lichtlen P, Georgiev O, Schaffner W. Characterization of the mouse gene for the heavy metal-responsive transcription factor MTF-1. Cell Stress Chaperones. 2000;5:196-206.

Banni M, Hajer A, Sforzini S, Oliveri C, Boussetta H, Viarengo A. Transcriptional expression levels and biochemical markers of oxidative stress in Mytilus galloprovincialis exposed to nickel and heat stress. Comp Biochem Physiol C Pharmacol Toxicol Endocrinol. 2014;160:23-9.

Bi Y, Lin GX, Millecchia L, Ma Q. Superinduction of metallothionein I by inhibition of protein synthesis: role of a labile repressor in MTF-1 mediated gene transcription. J Biochem Mol Toxicol. 2006;20:57-68.

Bittel DC, Smirnova IV, Andrews GK. Functional heterogeneity in the zinc fingers of metalloregulatory protein metal response element-binding transcription factor-1. J Biol Chem. 2000;275:37194-201.

Chen X, Chu M, Giedroc DP. MRE-Binding transcription factor-1: weak zinc-binding finger domains 5 and 6 modulate the structure, affinity, and specificity of the metal-response element complex. Biochemistry. 1999;38:12915-25.

Chen W-Y, John JAC, Lin C-H, Chang C-Y. Molecular cloning and developmental expression of zinc finger transcription factor MTF-1 gene in zebrafish, Danio rerio. Biochem Biophys Res Commun. 2002;291:798-805.

Chen W, John JAC, Lin C, Chang C. Expression pattern of metallothionein, MTF-1 nuclear translocation, and its dna-binding activity in zebrafish (Danio rerio) induced by zinc and cadmium. Environ Toxicol Chem. 2007;26:110-7.

Cheuk WK, Chan PC-Y, Chan KM. Cytotoxicities and induction of metallothionein (MT) and metal regulatory element (MRE)-binding transcription factor-1 (MTF-1) messenger RNA levels in the zebrafish (Danio rerio) ZFL and SJD cell lines after exposure to various metal ions. Aquat Toxicol. 2008;89:103-12.

Cheung AP-L, Au CY-M, Chan WW-L, Chan KM. Characterization and localization of metal-responsive-element-binding transcription factors from tilapia. Aquat Toxicol. 2010;99:42-55.

Cho YS, Lee SY, Bang IC, Kim DS, Nam YK. Genomic organization and mRNA expression of manganese superoxide dismutase (Mn-SOD) from Hemibarbus mylodon (Teleostei, Cypriniformes). Fish Shellfish Immunol. 2009;27:571-6.

De SK, Enders GC, Andrews GK. High levels of metallothionein messenger RNAs in male germ cells of the adult mouse. Mol Endocrinol. 1991:5:628-36.

Dubé A, Harrisson J-F, Saint-Gelais G, Séguin C. Hypoxia acts through multiple signaling pathways to induce metallothionein transactivation by the metal-responsive transcription factor-1 (MTF-1). Biochem Cell Biol. 2011;89:562-77.

Ferencz Á, Hermesz E. Identification and characterization of two mtf-1 genes in common carp. Comp Biochem Physiol C Toxicol Pharmacol. 2008;148:238-43.
Ferencz Á, Hermesz E. Identification of a splice variant of the metal-responsive transcription factor MTF-1 in common carp. Comp Biochem Physiol C Toxicol Pharmacol. 2009;150:113-7.

Giedroc DP, Chen X, Apuy JL. Metal response element (MRE)-binding transcription factor-1 (MTF-1): structure, function, and regulation. Antioxid Redox Signal. 2001;3:577-96.

Günes C, Heuchel R, Georgiev O, Müller K-H, Lichtlen P, Blüthmann H, Marino S, Aguzzi A, Schaffner W. Embryonic lethality and liver degeneration in mice lacking the metal-responsive transcriptional activator MTF-1. EMBO J. 1998;17:2846-54.

Günther V, Lindert U, Schaffner W. The taste of heavy metals: Gene regulation by MTF-1. Biochim Biophys Acta, Mol Cell Res. 2012a;1823:1416-25.

Günther V, Davis AM, Georgiev O, Schaffner W. A conserved cysteine cluster, essential for transcriptional activity, mediates homodimerization of human metal-responsive transcription factor-1 (MTF-1). Biochim Biophys Acta, Mol Cell Res. 2012b;1823:476-83.

Hahn ME, Karchner SI, Evans BR, Franks DG, Merson RR, Lapseritis JM. Unexpected diversity of aryl hydrocarbon receptors in non-mammalian vertebrates: insights from comparative genomics. J Exp Zool A Ecol Genet Physiol. 2006;305:693-706.

Jackson DP, Joshi AD, Elferink CJ. Ah receptor pathway intricacies; signaling through diverse protein partners and DNA-motifs. Toxicol Res. 2015;4:1143-58.

Jarque S, Prats E, Olivares A, Casado M, Ramón M, Piña B. Seasonal variations of gene expression biomarkers in Mytilus galloprovincialis cultured populations: temperature, oxidative stress and reproductive cycle as major modulators. $\mathrm{Sci}$ Total Environ. 2014;499:363-72.

Jenny MJ, Warr GW, Ringwood AH, Baltzegar DA, Chapman RW. Regulation of metallothionein genes in the American oyster (Crassostrea virginica): ontogeny and differential expression in response to different stressors. Gene. 2006;379:156-65.

Kang J, Lee YG, Jeong DU, Lee JS, Choi YH, Shin YK. Effect of abalone farming on sediment geochemistry in the Shallow Sea near Wando, South Korea. Ocean Sci J. 2015;50:669-82

Kim K-Y, Lee SY, Cho YS, Bang IC, Kim KH, Kim DS, Nam YK. Molecular characterization and mRNA expression during metal exposure and thermal stress of copper/zinc-and manganese-superoxide dismutases in disk abalone, Haliotis discus discus. Fish Shellfish Immunol. 2007;23:1043-59.

Laity JH, Andrews GK. Understanding the mechanisms of zinc-sensing by metal-response element binding transcription factor-1 (MTF-1). Arch Biochem Biophys. 2007;463:201-10.

Lee SY, Nam YK. Transcriptional responses of metallothionein gene to different stress factors in Pacific abalone (Haliotis discus hannai). Fish Shellfish Immunol. 2016a:58:530-41.

Lee SY, Nam YK. Evaluation of reference genes for RT-qPCR study in abalone Haliotis discus hannai during heavy metal overload stress. Fish Aquat Sci. 2016b;19:21

Li Y, Kimura T, Laity JH, Andrews GK. The zinc-sensing mechanism of mouse MTF-1 involves linker peptides between the zinc fingers. Mol Cell Biol. 2006:26:5580-7.

Li Y, Kimura T, Huyck RW, Laity JH, Andrews GK. Zinc-induced formation of a coactivator complex containing the zinc-sensing transcription factor MTF-1, p300/CBP, and Sp1. Mol Cell Biol. 2008;28:4275-84.

Lichtlen P. Schaffner W. Putting its fingers on stressful situations: the heavy metal-regulatory transcription factor MTF-1. BioEssays. 2001;23:1010-7.

Lichtlen P, Wang Y, Belser T, Georgiev O, Certa U, Sack R, Schaffner W. Target gene search for the metal-responsive transcription factor MTF-1. Nucleic Acids Res. 2001;29:1514-23.

Mao H, Wang D-H, Yang W-X. The involvement of metallothionein in the development of aquatic invertebrate. Aquat Toxicol. 2012;110:208-13.

Meng J, Zhang L, Li L, Li C, Wang T, Zhang G. Transcription factor CgMTF-1 regulates CgZnT1 and CgMT expression in Pacific oyster (Crassostrea gigas) under zinc stress. Aquat Toxicol. 2015;165:179-88.

Negri A, Oliveri C, Sforzini S, Mignione F, Viarengo A, Banni M. Transcriptional response of the mussel Mytilus galloprovincialis (Lam.) following exposure to heat stress and copper. PLoS One. 2013;8:e66802.

O'Shields B, McArthur AG, Holowiecki A, Kamper M, Tapley J, Jenny MJ. Inhibition of endogenous MTF-1 signaling in zebrafish embryos identifies novel roles for MTF-1 in development. Biochim Biophys Acta, Mol Cell Res. 2014:1843:1818-33.

Park C-J, Kim SY. Abalone aquaculture in Korea. J Shellfish Res. 2013;32:17-9.

Qiu J, Liu Y, Yu M, Pang Z, Chen W, Xu Z. Identification and functional characterization of MRE-binding transcription factor (MTF) in Crassostrea 
gigas and its conserved role in metal-induced response. Mol Biol Rep. 2013;40:3321-31.

Roesijadi G, Hansen KM, Unger ME. Cadmium-induced metallothionein expression during embryonic and early larval development of the mollusc Crassostrea virginica. Toxicol Appl Pharmacol. 1996;140:356-63.

Saydam N, Georgiev O, Nakano MY, Greber UF, Schaffner W. Nucleo-cytoplasmic trafficking of metal-regulatory transcription factor 1 is regulated by diverse stress signals. J Biol Chem. 2001;276:25487-95.

Saydam N, Steiner F, Georgiev O, Schaffner W. Heat and heavy metal stress synergize to mediate transcriptional hyperactivation by metal-responsive transcription factor MTF-1. J Biol Chem. 2003;278:31879-83.

Schmittgen TD, Livak KJ. Analyzing real-time PCR data by the comparative CT method. Nat Protoc. 2008:3:1101-8.

Smirnova IV, Bittel DC, Ravindra R, Jiang H, Andrews GK. Zinc and cadmium can promote rapid nuclear translocation of metal response element-binding transcription factor-1. J Biol Chem. 2000;275:9377-84.

Stoytcheva ZR, Vladimirov V, Douet V, Stoychev I, Berry MJ. Metal transcription factor-1 regulation via MREs in the transcribed regions of selenoprotein $\mathrm{H}$ and other metal-responsive genes. Biochim Biophys Acta, Gen Subj. 2010;1800:416-24.

Uenishi R, Gong P, Suzuki K, Koizumi S. Cross talk of heat shock and heavy metal regulatory pathways. Biochem Biophys Res Commun. 2006;341:1072-7.

Wang $Y$, Wimmer $U$, Lichtlen $P$, Inderbitzin D, Stieger B, Meier PJ, Hunziker $L$, Stallmach T, Forrer R, Rulicke T, Georgiev O, Schaffner W. Metal-responsive transcription factor-1 (MTF-1) is essential for embryonic liver development and heavy metal detoxification in the adult liver. FASEB J. 2004;18:1071-9.

Zhang B, Egli D, Georgiev O, Schaffner W. The Drosophila homolog of mammalian zinc finger factor MTF-1 activates transcription in response to heavy metals. Mol Cell Biol. 2001;21:4505-14.

\section{Submit your next manuscript to BioMed Central and we will help you at every step:}

- We accept pre-submission inquiries

- Our selector tool helps you to find the most relevant journal

- We provide round the clock customer support

- Convenient online submission

- Thorough peer review

- Inclusion in PubMed and all major indexing services

- Maximum visibility for your research

Submit your manuscript at www.biomedcentral.com/submit

C) Biomed Central 